\title{
Efeito da calagem, do fosfato natural de Gafsa e superfosfato triplo no crescimento inicial e absorção de fósforo em Eucalyptus dunnii
}

\author{
Shizuo Maeda ${ }^{1}$, Itamar Antonio Bognola ${ }^{1}$ \\ ${ }^{1}$ Embrapa Florestas, Estrada da Ribeira, Km 411, CP 319, CEP 83411-000, Colombo, PR, Brasil
}

"Autor correspondente:

maeda@cnpf.embrapa.br

Termos para indexação:

Fontes de fósforo

Calagem

Fertilidade do solo

Index terms:

Phosphorus sources

Liming

Soil fertility

Histórico do artigo:

Recebido em 26 jul 2011

Aprovado em $01 \mathrm{dez} 2011$

Publicado em 28 dez 2011

doi: $10.4336 / 2011 . p f b .31 .68 .355$
Resumo - O efeito da aplicação combinada de calagem, fontes de fósforo e forma de aplicação das fontes no crescimento e absorção de fósforo em mudas de Eucalyptus dunnii foi estudado em condições de vaso. $\mathrm{O}$ experimento foi conduzido em casa-devegetação não climatizada, utilizando um Cambissolo Húmico Ta Alumínico Típico, durante 67 dias. Os tratamentos consistiram na combinação de dois níveis de calagem ( $\mathrm{C} 0$, sem calagem e $\mathrm{C} 1$, dose para elevar o $\mathrm{pH}$ em água a 5,5 pelo método do $\mathrm{pH} \mathrm{SMP}$ ), duas fontes de fósforo (superfosfato triplo e fosfato natural de Gafsa) e dois modos de aplicação das fontes de fósforo (incorporada e localizada). A calagem aumentou a produção de matéria seca da parte aérea, o crescimento das mudas, o acúmulo e a eficiência de uso do P no eucalipto. A aplicação incorporada de $\mathrm{P}$ foi mais eficiente que a localizada. A calagem diminuiu eficiência do fosfato natural de Gafsa, e o superfosfato triplo foi a fonte mais eficiente.

\section{Effect of liming, Gafsa rock phosphate and triple} superphosphate in early growth and phosphorus uptake in Eucalyptus dunnii

\begin{abstract}
The effect of combined application of lime, phosphorus sources and forms of application of the phosphorus sources on growth and P uptake of Eucalyptus dunnii have been studied in pots. The treatments consisted of two levels of liming $(\mathrm{C} 0, \mathrm{C} 1$ lime to raise to $\mathrm{pH} 5.5$ in water by $\mathrm{pH}$ SMP method), two phosphorus sources (triple superphosphate and Gafsa rock phosphate) and two forms of application of phosphorus sources (incorporated and located). The experiment was carried out in a greenhouse using a Humic Cambisol Ta aluminate Typical for 67 days. Liming increased dry matter production of shoots, plant growth, accumulation and P use efficiency in Eucalyptus; the incorporated application was more efficient than localized. Liming decreased efficiency of Gafsa rock phosphate and triple superphosphate was the most efficient phosphorus source.
\end{abstract}

\section{Introdução}

No Brasil, os plantios florestais são realizados predominantemente em solos de baixa fertilidade natural, que são altamente intemperizados e apresentam elevada capacidade de fixação de fósforo. Nesse ambiente, as estratégias de manejo da fertilidade do solo para o aumento da disponibilidade do fósforo para as plantas podem se valer da escolha de fontes mais adequadas do nutriente ou pela calagem, entre outras (Novais \& Smyth, 1999). 
Os fosfatos naturais disponíveis no Brasil são, em sua maioria, de baixa solubilidade e apresentam concentrações de fósforo relativamente baixa, apresentando baixa eficiência inicial e desestimulando sua utilização direta. Entre as fontes de fósforo mais utilizadas no Brasil destaca-se o superfosfato triplo, que se caracteriza pela alta solubilidade e elevado teor do nutriente disponível para as plantas $\left(45 \%\right.$ de $\mathrm{P}_{2} \mathrm{O}_{5}$ determinado em ácido cítrico a $2 \%$ ), sendo obtido pela solubilização ácida de fosfatos de rocha de baixa solubilidade (Raij et al., 1982).

Fosfatos naturais reativos, como é o caso do fosfato natural de Gafsa (hiperfosfato), têm sido utilizados pela maior eficiência em relação às fontes nativas do Brasil. Esse produto pode apresentar eficiência semelhante aos superfosfatos quando apresenta granulometria fina e incorporado ao solo na camada superficial. Contudo, sua eficiência é diminuída quando aplicada de forma localizada (Raij et al., 1982).

A difusão é a forma mais importante de absorção de fósforo pelas plantas, sendo facilitado quando estas apresentam um sistema radicular bem desenvolvido e uma boa distribuição do nutriente no perfil do solo. A aplicação localizada de fósforo tem a vantagem de limitar temporariamente a fixação do elemento, porém, ocorre a redução do contato das raízes com o nutriente, resultando em menor absorção e restrição no crescimento da planta. Por outro lado, quanto melhor a distribuição do fósforo aplicado ao solo, maior será o seu contato e, por consequência, maior será a sua adsorção, resultando em menor disponibilidade para as plantas (Novais \& Smith, 1999).

Em condições de solo ácido, os óxidos de alumínio e ferro são os principais elementos responsáveis pela fixação do fósforo (JORGE, 1980). Em solos ricos em matéria orgânica, a adsorção de fósforo é reduzida, sendo esse efeito atribuído ao bloqueio dos sítios de adsorção (ALMEIDA et al., 2003; ARAÚJO et al., 2004; SOUZA et al., 2006). Conforme TRAINA et al. (1986), ácidos orgânicos do solo solubilizam fosfatos de ferro e alumínio, reduzindo a sua precipitação com íons de ferro e alumínio e diminuem a adsorção de fósforo por oxidróxidos de ferro e de alumínio. Estes minerais podem adsorver as substâncias húmicas com grande energia e assim competirem com o fosfato pelos mesmos sítios de adsorção (HAYNES, 1984).

O objetivo desse trabalho é comparar o efeito da calagem e da forma de aplicação na eficiência de duas fontes de fósforo no crescimento e absorção do nutriente por mudas de Eucalyptus dunnii em solo com elevado teor de acidez trocável $\left(\mathrm{Al}^{+3}\right)$.

\section{Material e métodos}

O experimento foi conduzido em vaso, em condições de casa-de-vegetação não climatizada da Embrapa Florestas, em Colombo, PR, no período de fevereiro a abril de 2011, totalizando 67 dias. Foram utilizadas mudas de Eucalyptus dunnii obtidas por estaquia. As mudas foram conduzidas em tubetes de $50 \mathrm{~mL}$ em substrato comercial durante 90 dias, sendo em seguida transplantadas para vasos plásticos com capacidade de $4 \mathrm{~L}$, dando início ao estudo.

Foi utilizado um Cambissolo Húmico Ta Alumínico Típico (SANTOS et al., 2006), coletado em Rio Negrinho, SC, anteriormente cultivado com pínus, e submetido ao corte raso para plantio de eucalipto. A amostra de solo utilizada foi coletada na camada de 0 a $20 \mathrm{~cm}$ de profundidade. $\mathrm{O}$ seu preparo constou da secagem ao ar e passagem em peneira com malha de $5 \mathrm{~mm}$. As características químicas e físicas, analisadas segundo Silva (1999), apresentaram os seguintes valores: $\mathrm{pH} \mathrm{SMP}=4,30 ; \mathrm{pH} \mathrm{CaCl}_{2}=4,12 ; \mathrm{P}($ Mehlich-1 $)=1,38$ $\mathrm{mg} \mathrm{dm}{ }^{-3} ; \mathrm{K}^{+}=0,10 \mathrm{cmol}_{\mathrm{c}} \mathrm{dm}^{-3} ; \mathrm{Ca}^{+2}=0,03 \mathrm{cmol}_{\mathrm{c}} \mathrm{dm}^{-3}$; $\mathrm{Mg}^{+2}=0,01 \mathrm{cmol}_{\mathrm{c}} \mathrm{dm}^{-3} ; \mathrm{Al}^{+3}=9,24 \mathrm{cmol}_{\mathrm{c}} \mathrm{dm}^{-3} ; \mathrm{H}+\mathrm{Al}$ $=25,3 \mathrm{cmol}_{\mathrm{c}} \mathrm{dm}^{-3} ; \mathrm{T}=25,44 \mathrm{cmol}_{\mathrm{c}} \mathrm{dm}^{-3} ; \mathrm{V}=2,0 \% ; \mathrm{m}$ $=94 \%$; matéria orgânica $=54,7 \mathrm{~g} \mathrm{~kg}^{-1}$; argila $=35,5 \%$; areia grossa $=1,0 \%$; areia fina $=8,5 \%$ e silte $=54,0 \%$.

Os tratamentos consistiram na combinação de dois níveis de calagem $(\mathrm{C} 0$, sem calagem e $\mathrm{C} 1$, calagem para elevar o $\mathrm{pH}$ em água a 5,5 pelo método do $\mathrm{pH}$ SMP, conforme THOMÉ JUNIOR, 1997), duas fontes de fósforo (superfosfato triplo - SFT e fosfato natural de Gafsa - GAF) na dose $200 \mathrm{mg}$ de $\mathrm{P} \mathrm{kg}^{-1}$ de solo e duas formas de aplicação de fósforo (incorporado ao solo e localizado). O SFT continha $42 \%$ de $\mathrm{P}_{2} \mathrm{O}_{5}$ solúvel em água e $3 \%$ de $\mathrm{P}_{2} \mathrm{O}_{5}$ solúvel em citrato neutro de amônio, e o GAF continha $28 \%$ de $\mathrm{P}_{2} \mathrm{O}_{5}$ solúvel em ácido cítrico. $\mathrm{O}$ experimento foi conduzido no esquema fatorial $2 \times 2 \times 2$ (dois níveis de calagem, duas fontes de fósforo e dois modos de aplicação) em delineamento inteiramente casualizado, com três repetições, sendo a unidade experimental constituída por um vaso com uma planta.

As doses de corretivos $\left(\mathrm{CaCO}_{3}\right.$ e $\mathrm{MgCO}_{3}$ p.a., relação estequiométrica de $4: 1$ ) de cada tratamento foram misturadas a $3,4 \mathrm{~kg}$ de terra e incubadas durante 
dez dias em umidade correspondente à capacidade de campo. Após a incubação, as amostras do solo foram secas para a aplicação das doses das fontes de fósforo correspondentes a cada tratamento, sendo a aplicação do tratamento incorporado realizado de forma homogênea em todo material experimental de cada parcela. $\mathrm{Na}$ aplicação localizada, feita com o auxílio de um funil, as doses dos produtos foram colocadas em dois orifícios com cerca de $3 \mathrm{~cm}$ de diâmetro e $10 \mathrm{~cm}$ de profundidade, distantes $5 \mathrm{~cm}$ da cova de plantio da muda. O plantio das mudas foi realizado após a aplicação do tratamento em que as fontes de $\mathrm{P}$ foram incorporadas. No caso do tratamento em que as fontes de fósforo foram aplicadas de forma localizada, o plantio das mudas foi feito antes da aplicação dos produtos, sendo que em ambos os casos o plantio das mudas ocorreu no mesmo dia. Em todos os tratamentos foi feita uma adubação básica com $300 \mathrm{mg}$ de $\mathrm{N} \mathrm{kg}^{-1}$ solo [CO(NH$)_{2}$ p.a.], $300 \mathrm{mg}$ de $\mathrm{K}_{2} \mathrm{O}$ (KCl p.a.), $30 \mathrm{mg} \mathrm{S} \mathrm{kg}^{-1}$ de solo [( $\left.\mathrm{NH}_{4}\right)_{2} \mathrm{SO}_{4}$ p.a.] e micronutrientes, $150 \mathrm{mg} \mathrm{kg}^{-1}$ de solo (FTE BR12), sendo essas doses consideradas suficientes para o atendimento da necessidade das mudas durante o período experimental. A aplicação dessas fontes de nutrientes foi realizada antes da aplicação das fontes de fósforo.

A umidade do solo nos vasos foi mantida a valores próximos a $90 \%$ da capacidade de campo com a aplicação de água deionizada.

Foi feita uma amostragem de solo de cada unidade experimental no início de condução do ensaio para avaliação do $\mathrm{pH}$ em água e do teor de fósforo segundo Silva et al., (1999).

O efeito dos tratamentos no crescimento das mudas foi avaliado pela produção de matéria seca da parte aérea (MSPA), incremento relativo em altura (INCR alt) - obtido pelas avaliações inicial e final do período de condução do ensaio, teor e acúmulo de fósforo na
MSPA e eficiência de utilização de fósforo (razão da relação entre a MSPA e o fósforo acumulado). A parte aérea foi coletada cortando o caule das mudas rente ao solo. Após descontaminação por meio de lavagem em água deionizada, o material coletado foi seco em estufa com circulação forçada a $65^{\circ} \mathrm{C}$ por dois dias, e em seguida avaliada a massa seca de cada amostra. Após essa etapa, o material coletado foi moído em moinho tipo Wiley, sendo em seguida determinado o teor de fósforo, conforme Silva et al., (1999). Os dados obtidos foram submetidos à análise de variância e ao teste de médias (Tukey, $\mathrm{p}<0,05$ ).

\section{Resultados e discussão}

\section{Matéria seca da parte aérea e incremento relativo das mudas de Eucalyptus dunnii em altura}

A calagem aumentou significativamente a produção de matéria seca da parte aérea (MSPA). O superfosfato triplo (SFT) promoveu maior produção de MSPA do que o fosfato natural de Gafsa (GAF) tanto aplicado de forma incorporada quanto localizada. O modo de aplicação das fontes de fósforo alterou a MSPA produzida pela aplicação do SFT e pelo GAF, sendo a aplicação incorporada mais eficiente do que a aplicação localizada (Tabela 1).

O crescimento das mudas de E. dunnii avaliado pelo incremento relativo em altura (INCR alt) foi significativamente influenciado pelo modo de aplicação e pela fonte de fósforo. $\mathrm{O}$ efeito da calagem foi observado na aplicação localizada do SFT, observando-se maior crescimento das mudas com a aplicação de calcário enquanto no caso da aplicação incorporada do GAF observou-se crescimento inferior com essa mesma aplicação (Tabela 2). 
Tabela 1. Matéria seca da parte aérea de mudas de Eucalyptus dunnii submetidas à calagem, fontes de fósforo e modos de aplicação das fontes de fósforo em Cambissolo Húmico Ta Alumínico Típico, durante 67 dias, em condições de vaso.

\begin{tabular}{|c|c|c|c|c|c|c|c|}
\hline \multirow{3}{*}{ Tratamentos } & \multicolumn{7}{|c|}{ Matéria seca da parte aérea $\left(g\right.$ planta $\left.^{-1}\right)$} \\
\hline & \multicolumn{3}{|c|}{ SFT } & \multicolumn{3}{|c|}{ GAF } & \multirow{2}{*}{$\begin{array}{c}\text { Média } \\
\text { calagem }\end{array}$} \\
\hline & INC & LOC & $\begin{array}{c}\text { Média } \\
\text { calagem }\end{array}$ & INC & LOC & $\begin{array}{c}\text { Média } \\
\text { calagem }\end{array}$ & \\
\hline $\mathrm{C} 0$ & $11,58 \mathrm{aB}$ & $9,37 \mathrm{bB}$ & $10,45 \mathrm{~B}$ & $10,04 \mathrm{aB}$ & $6,42 \mathrm{bB}$ & $8,23 \mathrm{~B}$ & $9,35 \mathrm{~B}$ \\
\hline \multirow[b]{2}{*}{ Média fonte } & 14,51 aA & $13,94 \mathrm{aA}$ & \multirow{4}{*}{$14,22 \mathrm{~A}$} & $14,66 \mathrm{aA}$ & $9,96 \mathrm{bA}$ & \multirow{5}{*}{$12,31 \mathrm{~A}$} & \multirow{5}{*}{$13,67 \mathrm{~A}$} \\
\hline & \multicolumn{2}{|c|}{$12,35 \mathrm{a}$} & & \multicolumn{2}{|c|}{$10,27 \mathrm{~b}$} & & \\
\hline Média modo aplicação do SFT & $13,04 \mathrm{a}$ & $12,35 \mathrm{a}$ & & & & & \\
\hline Média modo aplicação do GAF & & & & $11,66 \mathrm{a}$ & $8,19 \mathrm{~b}$ & & \\
\hline Média geral modo aplicação & $12,70 \mathrm{a}$ & $9,92 \mathrm{~b}$ & & & & & \\
\hline
\end{tabular}

Ausência (C0), presença (C1); fontes de fósforo: superfosfato triplo (SFT), fosfato natural de Gafsa (GAF); incorporado (INC), localizado (LOC). Médias seguidas por letras minúsculas iguais nas linhas dentro de fontes de fósforo e modos de incorporação e maiúsculas nas colunas não diferem significativamente entre si (Duncan, $5 \%$ ).

Tabela 2. Incremento relativo em altura (INCR alt) de mudas de Eucalyptus dunnii submetidas à calagem, fontes de fósforo e modos de aplicação das fontes de fósforo em Cambissolo Húmico Ta Alumínico Típico, durante 67 dias, em condições de vaso.

\begin{tabular}{|c|c|c|c|c|c|c|c|}
\hline \multirow{3}{*}{ Tratamentos } & \multicolumn{7}{|c|}{ INCR alt (\%) } \\
\hline & \multicolumn{3}{|c|}{ SFT } & \multicolumn{3}{|c|}{ GAF } & \multirow{2}{*}{$\begin{array}{c}\text { Média } \\
\text { calagem }\end{array}$} \\
\hline & INC & LOC & $\begin{array}{c}\text { Média } \\
\text { calagem }\end{array}$ & INC & LOC & $\begin{array}{c}\text { Média } \\
\text { calalgem }\end{array}$ & \\
\hline $\mathrm{c} 0$ & $110,51 \mathrm{aA}$ & $85,45 \mathrm{bB}$ & $97,98 \mathrm{~B}$ & $130,24 \mathrm{aA}$ & $83,12 \mathrm{bA}$ & $106,68 \mathrm{~A}$ & $102,33 \mathrm{~A}$ \\
\hline $\mathrm{c} 1$ & $131,54 \mathrm{aA}$ & $131,11 \mathrm{aA}$ & $131,32 \mathrm{~A}$ & $99,98 \mathrm{aB}$ & $84,16 \mathrm{aA}$ & $92,07 \mathrm{~A}$ & $111,70 \mathrm{~A}$ \\
\hline Média fonte & \multicolumn{2}{|c|}{$114,65 \mathrm{a}$} & & \multicolumn{2}{|c|}{$99,37 \mathrm{~b}$} & & \\
\hline Média modo aplicação do SFT & $121,03 \mathrm{a}$ & $108,28 \mathrm{a}$ & & & & & \\
\hline Média modo aplicação do GAF & & & & $115,11 \mathrm{a}$ & $83,64 \mathrm{~b}$ & & \\
\hline Média geral modo aplicação & $118,09 \mathrm{a}$ & $95,96 \mathrm{~b}$ & & & & & \\
\hline
\end{tabular}

Ausência (C0), presença (C1); fontes de fósforo: superfosfato triplo (SFT), fosfato natural de Gafsa (GAF); incorporado (INC), localizado (LOC).

Médias seguidas por letras minúsculas iguais nas linhas dentro de fontes de fósforo e modos de incorporação e maiúsculas nas colunas não diferem significativamente entre si (Duncan, $5 \%$ ).

O solo utilizado no estudo apresenta elevados teores de $\mathrm{Al}^{+3}$ e de matéria orgânica e baixos teores de $\mathrm{Ca}^{+2}$, $\mathrm{Mg}^{+2}$ e P. As espécies de Eucalyptus são consideradas tolerantes à acidez e à presença de $\mathrm{Al}^{+3}$, sendo, no entanto, exigentes quanto aos teores de $\mathrm{Ca}^{+2}, \mathrm{Mg}^{+2} \mathrm{e}$ P (Bellote \& Neves, 2001). Os teores considerados adequados para o $\mathrm{Ca}^{+2}$ e o $\mathrm{Mg}^{+2}$ são de 1,5 e $0,5 \mathrm{cmol}_{\text {c }}$ $\mathrm{dm}^{-3}$, respectivamente (Bellote \& Neves, 2001). A ausência de calagem acarretou na menor produção de MSPA, que pode ocorrer devido à carência de cálcio e de magnésio. Essa limitação atenuada pela calagem, o que resultou em maior produção de MSPA (Tabela 1).

A maior produção de MSPA obtida com a aplicação do SFT quando incorporado, comparado ao GAF (Tabela 1), pode dever-se à maior solubilidade do SFT, o que permitiu maior disponibilidade de fósforo às plantas, apesar da acidez do solo $\left(\mathrm{pH} \mathrm{CaCl}_{2}=4,2\right)$ e elevado teor de $\mathrm{Al}^{+3}\left(\mathrm{Al}^{+3}=9,24 \mathrm{cmol}_{\mathrm{c}} \cdot \mathrm{dm}^{-3}\right)$. É provável que o elevado teor de matéria orgânica presente no solo $\left(54,7\right.$ g. $\left.\mathrm{kg}^{-1}\right)$ utilizado no estudo tenha contribuído para minimizar a ação do $\mathrm{Al}^{+3}$ na adsorção do $\mathrm{P}$ liberado pelas fontes estudadas. $\mathrm{O}$ efeito da matéria orgânica na redução da adsorção dos íons fosfatos é atribuído ao bloqueio dos sítios de adsorção (Almeida et al., 2003; Araújo et al., 2004; Souza et al., 2006). No caso da ausência de efeito do modo de aplicação do SFT, é provável que a expansão das raízes tenha possibilitado o acesso ao fósforo aplicado mesmo de forma localizada. Ácidos orgânicos do solo solubilizam fosfatos de ferro e alumínio, reduzindo a sua precipitação com íons 
ferro e alumínio e diminuem a adsorção de fósforo por oxidróxidos de ferro e de alumínio (Traina et al., 1986). Estes metais podem se ligar a substâncias húmicas com grande energia e assim competirem com o fosfato pelos mesmos sítios de adsorção (Haynes, 1984). No caso do GAF, a aplicação incorporada favorece o contato do produto com o solo, o que aumenta a solubilização do mesmo, liberando maior quantidade do nutriente para as plantas quando comparado com a aplicação localizada (Raij et al., 1982). Condições ácidas do solo são favoráveis à solubilização dos fosfatos naturais, tendo em vista que expressiva proporção do fósforo neles contidos é solúvel em ácido cítrico ou citrato neutro de amônio diluídos, condições essas similares ao encontrado no solo utilizado $\left(\mathrm{pH} \mathrm{CaCl}{ }_{2}=4,12\right)$.

$\mathrm{O}$ maior incremento observado no crescimento das mudas com a incorporação das fontes de fósforo na ausência de calagem (Tabela 2) provavelmente devese também ao maior contato das raízes com o fósforo aplicado, uma vez que a difusão é a principal forma de absorção do elemento pelas plantas (Novais \& Smith, 1999).

É possível que o maior incremento observado no crescimento da mudas com a aplicação do superfosfato triplo na presença de calagem deva-se ao fornecimento de cálcio e de magnésio. Com relação ao fosfato natural de Gafsa, a calagem promoveu menor crescimento das mudas no caso da aplicação localizada, provavelmente pela redução na solubilização do produto em função da redução da acidez promovida pela calagem (Tabela 2)

\section{Teor e conteúdo de fósforo na parte aérea}

$O$ teor de fósforo na parte aérea das mudas de $E$. dunnii foi significativamente influenciado pelos fatores modo de aplicação das fontes estudadas, fonte de fósforo e pela calagem, ocorrendo aumento dos teores do fósforo com a aplicação de calcário e a incorporação das fontes de fósforo (Tabela 3).

Os teores de fósforo na matéria seca da parte aérea são resultantes da absorção do nutriente pelas plantas e do crescimento das plantas, podendo ocorrer a concentração do elemento com a absorção do mesmo sem o crescimento das plantas ou a sua diluição como resultado da baixa absorção com elevado crescimento. $\mathrm{Na}$ ausência de calagem, o teor de fósforo foi maior na aplicação localizada enquanto que com a aplicação do GAF não houve efeito do modo de aplicação do fósforo. Na presença da calagem, os teores de fósforo, tanto para SFT quanto para GAF, foi maior com a incorporação das fontes de fósforo (Tabela 3).

A calagem aumentou significativamente a quantidade de fósforo acumulado na parte aérea das mudas de $E$. dunnii. O superfosfato triplo promoveu maior acúmulo de fósforo na parte aérea das mudas da espécie estudada do que o fosfato natural de Gafsa. O modo de aplicação das fontes de fósforo aumentou a quantidade de fósforo acumulado pela aplicação do SFT e pelo GAF. A aplicação incorporada proporcionou maior teor e maior acúmulo de fósforo na parte aérea de mudas de $E$. dunnii na presença de calagem para ambos os produtos (Tabela 4).

Tabela 3. Teores de P na matéria seca da parte aérea de mudas de Eucalyptus dunnii submetidas à calagem, fontes de fósforo e modos de aplicação das fontes de fósforo em Cambissolo Húmico Ta Alumínico Típico, durante 67 dias, em condições de vaso.

\begin{tabular}{|c|c|c|c|c|c|c|c|}
\hline \multirow{3}{*}{ Tratamentos } & \multicolumn{7}{|c|}{ Teor de $\mathbf{P}\left(\mathrm{mg} \mathrm{kg}^{-1}\right)$} \\
\hline & \multicolumn{3}{|c|}{ SFT } & \multicolumn{3}{|c|}{ GAF } & \multirow{2}{*}{$\begin{array}{c}\text { Média } \\
\text { calagem }\end{array}$} \\
\hline & INC & LOC & $\begin{array}{c}\text { Média } \\
\text { calagem }\end{array}$ & INC & LOC & $\begin{array}{c}\text { Média } \\
\text { calagem }\end{array}$ & \\
\hline $\mathrm{C} 0$ & $1,08 \mathrm{bA}$ & $2,05 \mathrm{aA}$ & $1,56 \mathrm{~B}$ & $1,30 \mathrm{aB}$ & $1,07 \mathrm{aA}$ & $1,19 \mathrm{~B}$ & $1,38 \mathrm{~B}$ \\
\hline $\mathrm{C} 1$ & $2,27 \mathrm{aA}$ & $1,88 \mathrm{bA}$ & $2,08 \mathrm{~A}$ & $2,38 \mathrm{aA}$ & $1,03 \mathrm{bA}$ & $1,71 \mathrm{~A}$ & $1,89 \mathrm{~A}$ \\
\hline Média fonte & \multicolumn{2}{|c|}{$2,08 \mathrm{a}$} & & \multicolumn{2}{|c|}{$1,71 \mathrm{~b}$} & & \\
\hline Média modo aplicação do SFT & $1,68 \mathrm{~b}$ & $1,97 \mathrm{a}$ & & & & & \\
\hline Média modo aplicação do GAF & & & & $1,81 \mathrm{a}$ & $1,05 \mathrm{a}$ & & \\
\hline Média geral modo aplicação & $1,76 \mathrm{a}$ & $1,51 \mathrm{~b}$ & & & & & \\
\hline
\end{tabular}

Ausência (C0), presença (C1); fontes de fósforo: superfosfato triplo (SFT), fosfato natural de Gafsa (GAF); incorporado (INC), localizado (LOC).

Médias seguidas por letras minúsculas iguais nas linhas dentro de fontes de fósforo e modos de incorporação e maiúsculas nas colunas não diferem significativamente entre si (Duncan, 5\%). 
Tabela 4. Fósforo acumulado na parte aérea de mudas de Eucalyptus dunnii submetidas à calagem, fontes de fósforo e modos de aplicação das fontes de fósforo em Cambissolo Húmico Ta Alumínico Típico, durante 67 dias, em condições de vaso.

\begin{tabular}{|c|c|c|c|c|c|c|c|}
\hline \multirow{3}{*}{ Tratamentos } & \multicolumn{7}{|c|}{ Fósforo acumulado (mg planta ${ }^{-1}$ ) } \\
\hline & \multicolumn{3}{|c|}{ SFT } & \multicolumn{3}{|c|}{ GAF } & \multirow{2}{*}{$\begin{array}{c}\text { Média } \\
\text { calagem }\end{array}$} \\
\hline & INC & LOC & $\begin{array}{c}\text { Média } \\
\text { calagem }\end{array}$ & INC & LOC & $\begin{array}{c}\text { Média } \\
\text { calagem }\end{array}$ & \\
\hline $\mathrm{C} 0$ & $12,52 \mathrm{bB}$ & $18,88 \mathrm{aB}$ & $15,70 \mathrm{~B}$ & $13,21 \mathrm{Aa}$ & $6,86 \mathrm{bA}$ & $10,03 \mathrm{~B}$ & $12,86 \mathrm{~B}$ \\
\hline \multirow[b]{2}{*}{ Média fonte } & $33,01 \mathrm{aA}$ & $26,27 \mathrm{bA}$ & $29,64 \mathrm{~A}$ & $34,93 \mathrm{Ab}$ & $10,43 \mathrm{bA}$ & $22,68 \mathrm{~A}$ & $26,16 \mathrm{~A}$ \\
\hline & \multicolumn{2}{|c|}{$22,67 \mathrm{a}$} & & \multicolumn{2}{|c|}{$16,36 \mathrm{~b}$} & & \\
\hline Média modo aplicação do SFT & $24,06 \mathrm{a}$ & $22,76 \mathrm{a}$ & & & & & \\
\hline Média modo aplicação do GAF & & & & 22,58 a & $8,64 \mathrm{a}$ & & \\
\hline Média geral modo aplicação & $23,41 \mathrm{a}$ & $15,16 \mathrm{~b}$ & & & & & \\
\hline
\end{tabular}

Ausência (C0), presença (C1); fontes de fósforo: superfosfato triplo (SFT), fosfato natural de Gafsa (GAF); incorporado (INC), localizado (LOC).

Médias seguidas por letras minúsculas iguais nas linhas dentro de fontes de fósforo e modos de incorporação e maiúsculas nas colunas não diferem significativamente entre si (Duncan, $5 \%$ ).

\section{Eficiência de utilização de fósforo na parte aérea}

A eficiência de utilização do fósforo na parte aérea das mudas de E. dunnii foi significativamente influenciada pelos fatores fonte de fósforo e pela calagem, observandose redução da eficiência com a aplicação de calcário tanto do SFT quanto do GAF e menor eficiência do SFT comparado com o GAF (Tabela 5).
As maiores produções de MSPA foram obtidas nos tratamentos onde os teores, acúmulo e eficiência de utilização de fósforo foram confirmados pelos coeficientes de correlação positivos e significativos para as relações entre a MSPA e o teor de fósforo na parte aérea $(\mathrm{r}=0,70)$ a $5 \%$ de probabilidade, entre a MSPA e o conteúdo de fósforo $(\mathrm{r}=0,89)$, a $1 \%$ de probabilidade, e a MSPA e a eficiência de utilização do fósforo ( $\mathrm{r}=$ 0,70), também a $5 \%$.

Tabela 5. Eficiência de utilização do fósforo acumulado na parte aérea de mudas de Eucalyptus dunnii submetidas à calagem, fontes de fósforo e modos de aplicação das fontes de fósforo em Cambissolo Húmico Ta Alumínico Típico, durante 67 dias, em condições de vaso.

\begin{tabular}{|c|c|c|c|c|c|c|c|}
\hline \multirow{3}{*}{ Tratamentos } & \multicolumn{7}{|c|}{ Fósforo acumulado (mg planta $\left.{ }^{-1}\right)$} \\
\hline & \multicolumn{3}{|c|}{ SFT } & \multicolumn{3}{|c|}{ GAF } & \multirow{2}{*}{$\begin{array}{c}\text { Média } \\
\text { calagem }\end{array}$} \\
\hline & INC & LOC & $\begin{array}{c}\text { Média } \\
\text { calagem }\end{array}$ & INC & LOC & $\begin{array}{c}\text { Média } \\
\text { calagem }\end{array}$ & \\
\hline $\mathrm{C} 0$ & $0,93 \mathrm{aA}$ & $0,50 \mathrm{bA}$ & $0,71 \mathrm{~A}$ & $0,79 \mathrm{bA}$ & $0,96 \mathrm{aA}$ & $0,87 \mathrm{~A}$ & $0,79 \mathrm{~A}$ \\
\hline $\mathrm{C} 1$ & $0,44 \mathrm{aB}$ & $0,53 \mathrm{aA}$ & $0,48 \mathrm{~B}$ & $0,42 \mathrm{bB}$ & $0,98 \mathrm{aA}$ & $0,70 \mathrm{~B}$ & $0,59 \mathrm{~B}$ \\
\hline Média fonte & \multicolumn{2}{|c|}{$0,60 \mathrm{~b}$} & & \multicolumn{2}{|c|}{$0,79 \mathrm{a}$} & & \\
\hline Média modo aplicação do SFT & $0,68 \mathrm{a}$ & $0,51 \mathrm{~b}$ & & & & & \\
\hline Média modo aplicação do GAF & & & & $0,61 \mathrm{~b}$ & $0,97 \mathrm{a}$ & & \\
\hline Média geral modo aplicação & $0,64 \mathrm{a}$ & $0,74 \mathrm{a}$ & & & & & \\
\hline
\end{tabular}

Ausência (C0), presença (C1); fontes de fósforo: superfosfato triplo (SFT), fosfato natural de Gafsa (GAF); incorporado (INC), localizado (LOC).

Médias seguidas por letras minúsculas iguais nas linhas dentro de fontes de fósforo e modos de incorporação e maiúsculas nas colunas não diferem significativamente entre si (Duncan, $5 \%$ ).

\section{Conclusões}

A calagem aumentou a produção de matéria seca da parte aérea, o crescimento, o teor de fósforo e a quantidade de fósforo acumulado pelas mudas de Eucalyptus dunnii. Na ausência de calagem, a eficiência do superfosfato triplo e do fosfato natural de Gafsa na produção de matéria seca da parte aérea e no crescimento das mudas é maior na aplicação incorporada; na presença de calagem, o modo de aplicação não interfere no crescimento e na produção de matéria seca das mudas pela aplicação do superfosfato triplo. O superfosfato triplo é mais eficiente que o fosfato natural de Gafsa e a aplicação incorporada foi mais eficiente que a aplicação localizada. 


\section{Referências}

ALMEIDA, J. A.; TORRENT, J.; BARRÓN, V. Cor do solo, formas do fósforo e adsorção de fosfato em Latossolos desenvolvidos de basalto do extremo-sul do Brasil. Revista Brasileira de Ciência do Solo, Campinas, v. 27, p. 985-1002, 2003.

ARAÚJO, E. A.; LANI, J. L; AMARAL, E. F.; GUERRA, A. Uso da terra e propriedades físicas e químicas de Argissolo Amarelo Distrófico na Amazônia ocidental. Revista Brasileira de Ciência do Solo, Campinas, v. 28, p. 307-315, 2004.

BELLOTE, A. F. J.; NEVES, E. J. M. Calagem e adubação em espécies florestais plantadas na propriedade rural. Colombo: Embrapa Florestas, 2001. 6 p. (Embrapa Florestas. Circular Técnica, 54).

HAYNES, R. J. Lime and phosphate in the soil-plant system. Advances in Agronomy, New York, v. 37, p. 249-315, 1984.

JORGE, J. A. Fixação de fósforo em solos do estado de São Paulo. Bragantia, Campinas, v. 39, p. 99-113, 1980.

NOVAIS, R. F.; SMITH, T. J. Fósforo em solo e planta em condições tropicais. Viçosa: UFV/DPS, 1999. 399 p.

RAIJ, B. van; CABALA-ROSAND, P.; LOBATO, E. Adubação fosfatada no Brasil: apreciação geral, conclusões e recomendações. In: OLIVEIRA, A.; LOURENÇO, S.; GOEDERT, W.J. (Ed.). Adubação fosfatada no Brasil. Brasília, DF: EMBRAPA-DID, 1982. p. 9-28. (EMBRAPA-DID. Documentos, 21).
SANTOS, H. G. dos; JACOMINE, P. K. T.; ANJOS, L. H. C. dos; OLIVEIRA, V. A. de; OLIVEIRA, J. B. de; COELHO, M. R.; LUMBRERAS, J. F.; CUNHA, T. J. F. da (Ed.). Sistema brasileiro de classificação de solos. 2. ed. Rio de Janeiro: Embrapa Solos, 2006. 306 p.

SILVA, F. C. da (Org.). Manual de análises químicas de solos, plantas e fertilizantes. Brasília, DF: Embrapa Comunicação para Transferência de Tecnologia; Rio de Janeiro: Embrapa Solos; Campinas: Embrapa Informática Agropecuária, 1999. 370 p.

SOUZA, R. F.; FAQUIM, V.; TORRES, P. R. F.; BALIZA, D. P. Calagem e adubação orgânica: influência na adsorção de fósforo em solos. Revista Brasileira de Ciência do Solo, Campinas, v. 30, p. 975-983, 2006.

THOMÉ JÚNIOR, J. B. Manual para interpretação de análise de solo. Guaíba, RS: Livraria e Editora Agropecuária, 1997. $247 \mathrm{p}$.

TRAINA, S. J.; SPOSITO, G.; HESTERBERG, D.; KAFKAFI, $\mathrm{U}$. Effects of $\mathrm{pH}$ and organic acids on orthophosphate solubility in an acidic, montmorillonitic soil. Soil Science Society American Journal, Madison, v.50, p.45-52, 1986. 
